\title{
OBTENÇÃO DE PASTAS MINERAIS A PARTIR DO ESPESSAMENTO DE LAMAS PROVENIENTES DE PROCESSAMENTO FOSFÁTICO
}

\author{
M. C. REZENDE NETO*, K. C. FERREIRA, M. F. M. OLIVEIRA e G. E. S. VALADÃO \\ Universidade Federal de Minas Gerais \\ mariocrezende@yahoo.com.br*
}

Artigo submetido em novembro/2015 e aceito em dezembro/2015

DOI: $10.15628 /$ holos.2015.3706

\section{RESUMO}

Os minérios atualmente lavrados têm apresentado teores cada vez menores, impactando no aumento da produção de rejeitos e necessitando de técnicas para viabilizar sua disposição a seco. Entre essas técnicas, espessamento e processos de agregação de partículas, como a floculação, são utilizados para a produção de líquidos clarificados e de pastas minerais. Devido à menor quantidade de água, as pastas apresentam inúmeras vantagens ambientais e tecnológicas, sendo necessário considerar previamente suas propriedades reológicas, entre elas a altura de abatimento. 0 presente estudo objetivou caracterizar as pastas minerais obtidas a partir do espessamento de lamas, quanto ao seu abatimento, e selecionar a dosagem de floculante que apresente as melhores respostas de sedimentação e clarificação dos líquidos sobrenadantes. Os resultados foram obtidos através de ensaios de sedimentação descontínua em proveta, com floculante catiônico e aniônico, apresentando como única variável operacional a dosagem de floculante. Os líquidos clarificados obtidos em cada ensaio tiveram sua turbidez determinada por turbidímetro digital. No que se refere aos parâmetros reológicos, testes slump foram utilizados para determinação da consistência das pastas minerais, em ampla faixa de concentração de sólidos. Resultados mostraram que o floculante catiônico apresentou melhor resposta de sedimentação e clarificação de sobrenadante, cuja velocidade de sedimentação e turbidez foram, respectivamente, $2,40 \mathrm{~cm} / \mathrm{s}$ e $9,4 \mathrm{NTU}$. Para pastas com mais de $80 \%$ de sólidos, não foi observado abatimento significativo, próximo a $0,3 \mathrm{~cm}$, ao passo que, para polpas diluídas, as mesmas se espalhavam totalmente pela superfície.

PALAVRAS-CHAVE: pastas minerais, floculação, disposição superficial

\section{MINERAL PASTE RHEOLOGICAL CHARACTERISTIC OBTAINED FROM SLIMES THICKENING}

\section{ABSTRACT}

The ores currently mined have shown increasingly lower grades, impacting on increasing production of tailings and requiring techniques to enable their dry disposal. Among these techniques, thickening and particle aggregation processes, such as flocculation, have been used for production of clarified liquids and mineral pastes. Due to the smaller amount of water, the pastes have several environmental and technological advantages, but first it is necessary to consider their rheological properties, including the slump height. This study aims to characterize the mineral pastes obtained from the slimes thickening, as its slump height, in addition to select the flocculant dosage that presents the best settling answers and the best clarification of
\end{abstract}

the supernatants. The results were obtained by discontinuous settling tests in graduated cylinder with cationic and anionic flocculant, presenting as only operational variable the dosage of flocculant. The clarified liquids obtained in each test had their turbidity determined by digital turbidimeter. As rheological parameters, slump tests were used to determine the consistency of mineral pastes in wide concentration range of solids. The results showed that the cationic flocculant had better settling and clarification, whose settling rate and turbidity were, respectively, $2,40 \mathrm{~cm} / \mathrm{s}$ and 9,4 NTU. For pastes with more than $80 \%$ of solids, there was no significant slump, close to $0,3 \mathrm{~cm}$, while diluted slurries had total scattering over the surface

KEYWORDS: mineral pastes, flocculation, surface disposal 


\section{INTRODUÇÃO}

A mineração é uma atividade primordial ao homem, entretanto, o processamento de minérios gera um grande volume de resíduos que não apresentam valor econômico.

Como a lavra de minérios de menores teores metálicos tende a se tornar cada vez mais frequente, maiores volumes de rejeitos são produzidos, em diferentes granulometrias e provenientes de diferentes operações. Como a maior parte das operações de beneficiamento de minérios é realizada a úmido, os rejeitos geralmente se apresentam como polpas minerais e são dispostos em barragens, ainda que, de acordo com Sofrá e Boger (2002), existam potenciais riscos de rompimento, geração de drenagem ácida, contaminação de lençóis freáticos com reagentes, e difícil recuperação do solo e da vegetação original. Como grandes volumes de água não são reaproveitados, a disposição das polpas requer uma grande área de armazenamento.

Devido aos impactos ambientais ocasionados, a escassez dos recursos hídricos e a atual política de conservação de água, alternativas de disposição a seco se mostram vantajosas frente ao tradicional método de disposição de rejeitos úmidos. Para isso, o adensamento das polpas tem sido praticado, produzindo líquidos clarificados que retornam à atividade operacional, e fluidos com características típicas de pastas minerais, definidas por Araujo et. al. (2003) e Theriault et. al. (2001) como sistemas coloidais devidamente desaguados, de aspecto homogêneo, nos quais não ocorre a segregação granulométrica de partículas quando dispostos de forma suave sobre superfícies estáveis e que não apresentam drenagem significativa de água quando transportados por tubulações.

Devido à menor quantidade de água, as pastas podem ser empilhadas superficialmente ou preencher cavidades subterrâneas. Em conformidade com Lara (2011), no primeiro processo, conhecido como stacking, as pastas são distribuídas uniformemente ao longo da área, facilitando sua secagem e adensamento natural. Como benefícios adquiridos pela utilização da técnica, destaca-se menor risco de ruptura, menor área para disposição, maior recuperação de água, facilidade de recomposição da topografia original e reabilitação da área. No segundo processo, conhecido como backfill, cavidades subterrâneas são preenchidas com misturas de vários materiais, sendo o pastefill, método utilizado para o descarte de rejeitos, o mais utilizado. $\mathrm{Na}$ preparação da mistura, pequenas dosagens de aditivos são adicionadas à pasta mineral, o que, de acordo com Sofrá e Boger (2002), incrementam suas propriedades mecânicas. Dessa forma, maiores recuperações nas minas subterrâneas podem ser alcançadas.

Olcay (2008) defende que a distribuição granulométrica do sólido é um importante parâmetro para a obtenção e avaliação das características de pastas minerais, uma vez que influencia sua porosidade e sua resistência. De acordo com Falcucci (2007), as pastas geralmente podem ser obtidas a partir de uma ampla faixa granulométrica, ainda que um mínimo de $15 \%$ das partículas do material deva apresentar tamanho inferior a $20 \mu \mathrm{m}$, concordando com o exposto por Jung e Biswas (2002), em que certa quantidade de partículas finas seja necessária para obtenção e bombeamento das pastas.

Conforme Tão et. al. (2008), devido à granulometria finamente reduzida, as lamas presentes nos rejeitos são muito estáveis em suspensões aquosas e apresentam velocidade de sedimentação extremamente lenta. Como modo de reduzir o estado de dispersão e incrementar 
sua sedimentação natural, processos de agregação coloidal vêm sendo utilizados para o adensamento de polpas e a clarificação do líquido sobrenadante, em especial a floculação quando combinada com sistemas mecânicos. Segundo Guimarães (2010), polímeros orgânicos de elevados pesos moleculares e baseados na química do acrílico são os floculantes mais utilizados na indústria mineral. Entretanto, caso sejam superdosados em um determinado sistema, cadeias poliméricas podem se adsorver excessivamente nas superfícies minerais, cujas forças de repulsão entre cadeias resultam na dispersão das partículas.

Visando conhecer o comportamento de uma suspensão mineral frente à sedimentação, ensaios de sedimentação descontínua são realizados em provetas. A partir do mesmo, a curva de sedimentação é obtida, cuja inclinação, em um dado tempo, fornece a velocidade de sedimentação.

Para o transporte e disposição das pastas, propriedades reológicas devem ser analisadas, em especial a altura de abatimento, que denota a deformação sofrida pela pasta perante esforços de variadas origens, sendo influenciada, entre outros fatores, pela concentração de sólidos na pasta mineral.

O presente estudo objetiva caracterizar as pastas minerais obtidas a partir do espessamento de lamas, quanto ao seu abatimento, e selecionar a dosagem de floculante que apresente as melhores respostas de sedimentação e clarificação dos líquidos sobrenadantes.

\section{METODOLOGIA}

Foram utilizadas amostras de lamas provenientes do processamento de minérios fosfáticos da Vale Fertilizantes, em Araxá, coletadas no final do circuito, e já em condições de disposição final em barragens.

Para fins de estudo granulométrico, foi utilizado o granulômetro Cilàs, modelo 1064, no qual as partículas foram mantidas dispersas através de sistema de ultrassom. Para a determinação da composição mineralógica, foi utilizado o difratômetro de raios $X$, marca Phillips, com controlador modelo PW3710/31, gerador PW1830/40 e goniômetro PW3020/00. Quanto ao método de interpretação dos dados, foi utilizado o banco de dados PDF-2 Release 2010 do International Centre for Diffraction Data e o software X'Pert-APD. A radiação utilizada foi CuK.

Ensaios de sedimentação descontínua foram realizados em provetas de $1000 \mathrm{~cm}^{3}$, visando o conhecimento das velocidades de sedimentação de suspensões diluídas. A única variável operacional avaliada até a presente etapa do estudo foi a dosagem de floculante, mantendo fixo o pH da suspensão, em pH 9, e a quantidade de sólidos da polpa previamente preparada, em 10\% em massa. Ensaios foram realizados na presença de poliacrilamidas catiônicas e aniônicas de altos pesos moleculares, ambas fornecidas pela Clariant.

A turbidez do líquido sobrenadante foi analisada em turbidímetro digital microprocessado de bancada, modelo TB 1000, marca Tecnopon. As medições foram realizadas no overflow obtido nos ensaios de sedimentação descontínua.

Para a determinação da consistência das pastas minerais, o teste slump foi realizado através de um cilindro de PVC com diâmetro e altura iguais a $10 \mathrm{~cm}$. Em tais ensaios, o cilindro foi totalmente preenchido com o material até completar seu volume interno, e em seguida colocado 
sobre uma superfície plana. Após retirá-lo verticalmente para cima, verificou-se, com o auxílio de uma trena, a altura de abatimento.

\section{RESULTADOS E DISCUSSÃO}

A distribuição granulométrica da amostra estudada, por fração granulométrica, é apresentada graficamente na Figura 1, na forma de curva acumulada. A amostra é composta preponderantemente por lamas, com $d_{50}$ próximo a $6,10 \mu m$ e, portanto, apropriada para a preparação de pastas minerais, segundo a definição de Falcucci (2007). A análise ainda fornece alguns tamanhos típicos que caracterizam uma distribuição granulométrica, como os parâmetros $d_{10}$ e $d_{90}$, que representam, respectivamente, a granulometria de 10 e $90 \%$ das partículas. Para a amostra estudada, o $d_{10}$ e o $d_{90}$ obtidos foram $0,85 \mu \mathrm{m}$ e $21,96 \mu \mathrm{m}$, respectivamente.

O estudo de identificação mineralógica, realizado em difratômetro de raios $X$ e apresentado na Figura 2, revelou uma grande presença de minerais com grupos carbonatos, tais como calcita, em maior proporção, e dolomita, em menor proporção, embora ainda contenha pequenas quantidades de moscovita, caolinita, apatita e quartzo. Visando conhecer a ocorrência exata de apatita, análises via fluorescência de raios $\mathrm{X}$ e microscopia eletrônica de varredura serão realizadas em uma etapa posterior ao trabalho.

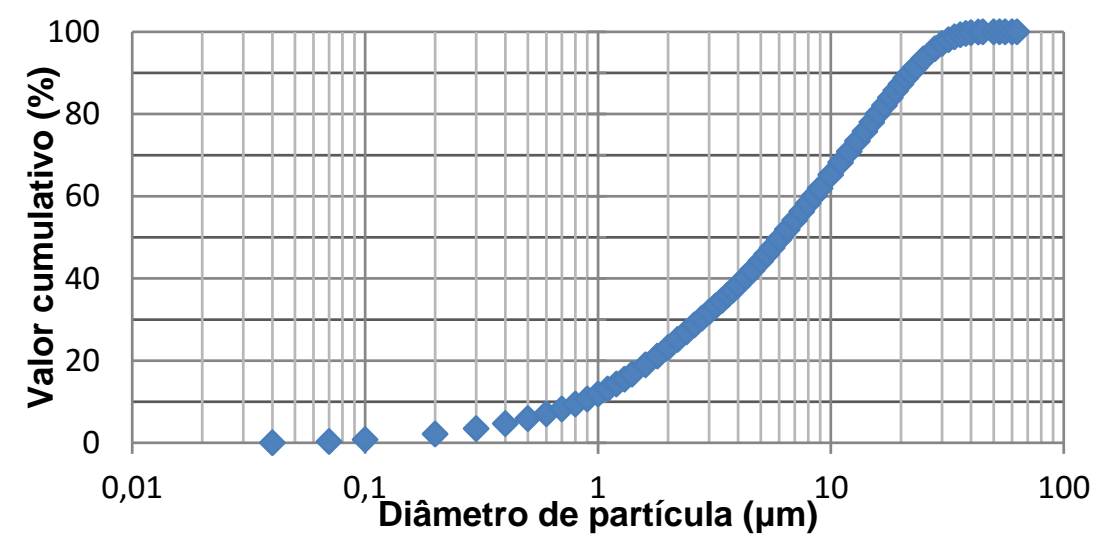

Figura 1: Curva de distribuição granulométrica acumulada.

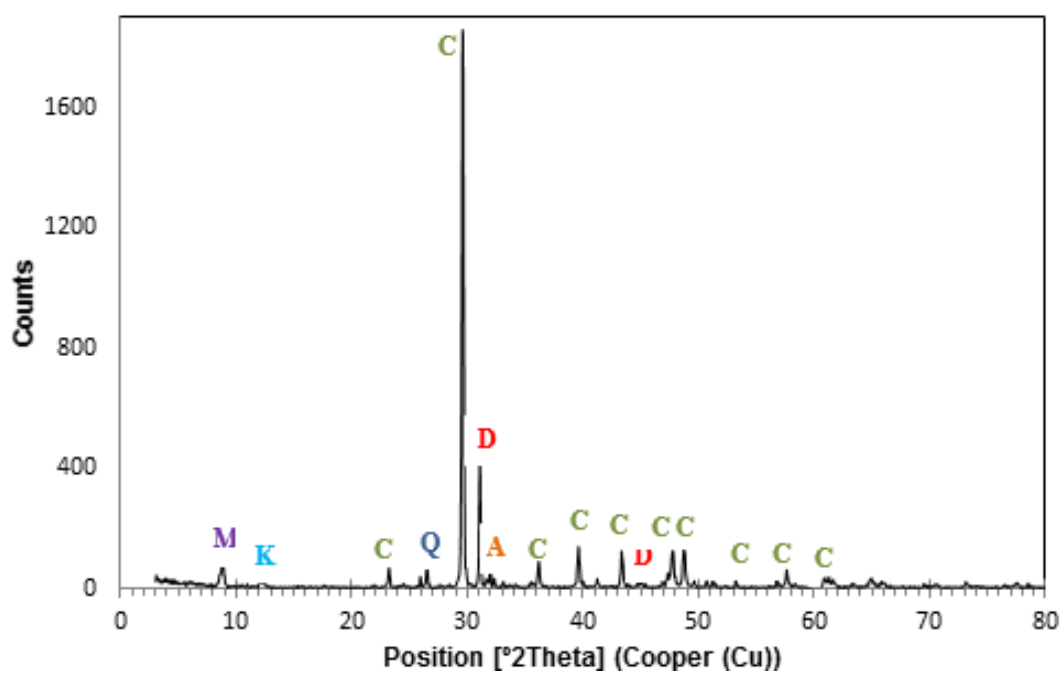

Figura 2: Difratograma de raios $\mathrm{X}$ da amostra estudada. Espécies: $\mathrm{M}=$ moscovita; $\mathrm{K}=$ caolinita; $\mathrm{C}=$ calcita; $\mathbf{Q}=$ quartzo; $\mathrm{D}$ = dolomita; $\mathrm{A}$ = apatita 
Os resultados de ensaios de sedimentação descontínua em provetas de $1000 \mathrm{~cm}^{3}$ e na presença de poliacrilamida catiônica (PAC) e aniônica (PAA) são mostrados na Figura 3 . As velocidades de sedimentação obtidas na presença dos floculantes, em toda a gama de dosagem analisada, foi demasiadamente superior em relação à suspensão mineral sem floculante $(0 \mathrm{~g} / \mathrm{t})$, ainda que ambas tenham apresentado comportamento semelhante. Nota-se uma pequena sobreposição das curvas de velocidade de sedimentação em dosagens mais baixas, na presença dos dois floculantes, embora a velocidade de sedimentação apresentada pela poliacrilamida aniônica tenha sido ligeiramente melhor, em especial na dosagem $100 \mathrm{~g} / \mathrm{t}$. Contraditoriamente ao esperado, para os floculantes analisados não foram determinadas as dosagens que resultariam na reestabilização do sistema, o que culminaria na queda da velocidade de sedimentação do mesmo, embora um aumento de turbidez do overflow tenha sido notado.

A Figura 4, referente à turbidez do overflow em função da dosagem de poliacrilamidas catiônicas (PAC) e aniônicas (PAA), mostra que em pequenas dosagens de floculantes, especificamente $50 \mathrm{~g} / \mathrm{t}$, ocorre uma redução satisfatória da turbidez do líquido sobrenadante, sendo mais eficaz o floculante catiônico para clarificação desse efluente. Na mesma dosagem, o overflow obtido com a poliacrilamida catiônica apresentou turbidez 6,3 NTU, ao passo que o overflow obtido com o floculante aniônico apresentou turbidez 25,0 NTU, ambos demasiadamente inferiores à turbidez obtida em sedimentação natural, na ausência de floculantes (104 NTU). Em dosagens maiores, acima de $200 \mathrm{~g} / \mathrm{t}$ para o floculante aniônico e $400 \mathrm{~g} / \mathrm{t}$ para o floculante catiônico, ocorreu um acréscimo notório da turbidez dos overflows, se tornando ainda maior em relação à mesma suspensão mineral na ausência de floculante. Apesar do floculante aniônico apresentar velocidade de sedimentação ligeiramente maior na dosagem $100 \mathrm{~g} / \mathrm{t}$, o floculante catiônico apresentou turbidez relativamente menor na mesma dosagem, indicando uma eficiente clarificação da fase aquosa.

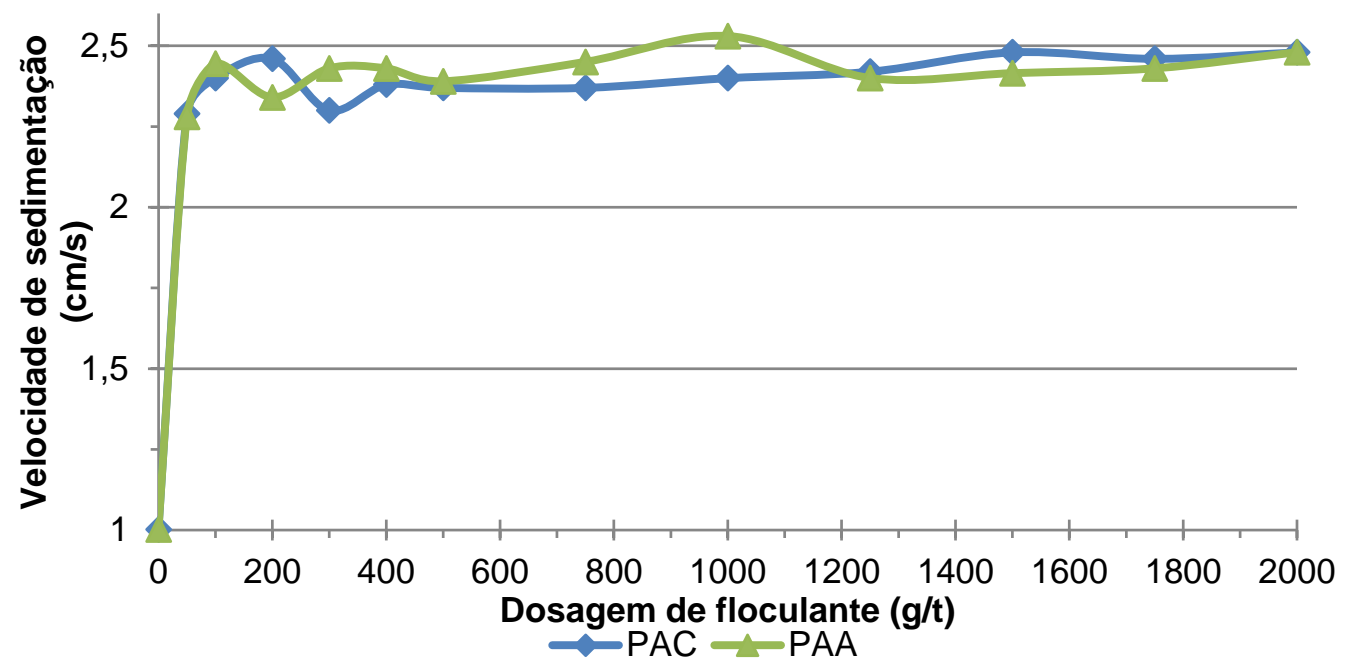

Figura 3: Velocidade de sedimentação com poliacrilamida catiônica (PAC) e aniônica (PAA).

Tal resposta pode ser justificada pelo excesso de sítios superficiais na superfície das lamas, determinado em um estudo preliminar via potencial Zeta. Dessa forma, interações eletrostáticas entre as superfícies minerais e o floculante catiônico se tornam mais eficazes que as interações com o floculante aniônico, aumentando a densidade de adsorção do primeiro e incrementando a sedimentação das partículas. Embora não tenha influenciado na sedimentação, dosagens maiores que $100 \mathrm{~g} / \mathrm{t}$ não se mostraram eficientes quanto à redução de turbidez, uma vez que, nessas 
dosagens, as cadeias poliméricas em excesso se repelem por apresentarem segmentos positivamente carregados ao longo de sua estrutura. Assim sendo, a melhor dosagem foi aquela em que se obteve apenas um recobrimento superficial das lamas, sem haver, no entanto, excesso de floculante.

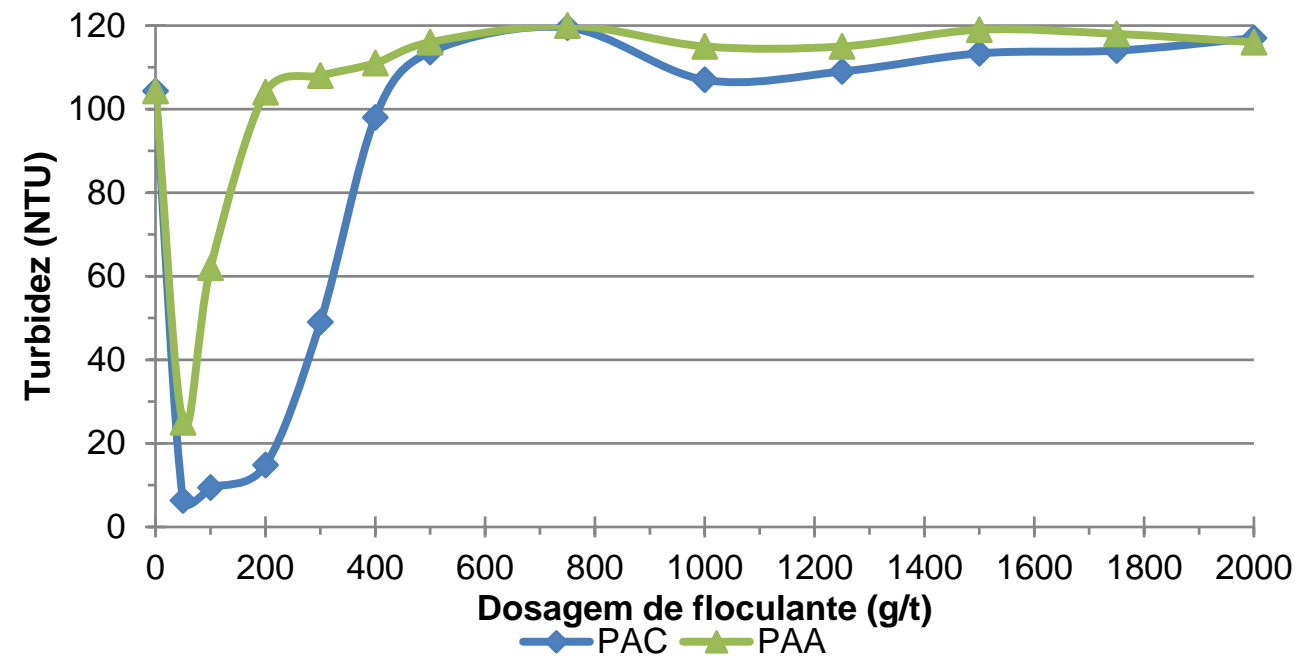

Figura 4. Turbidez do overflow com poliacrilamida catiônica (PAC) e aniônica (PAA).

Para avaliação do abatimento de pastas minerais obtidas artificialmente, foram utilizados testes slump, cujo resultado é apresentado na Figura 5. Como pode ser visto, com o aumento do desaguamento da polpa mineral, visando a produção de pastas, ocorre diminuição de seu abatimento, reduzindo assim a mobilidade de massa e aumentando a coesão entre as partículas minerais. Para a suspensão estudada, a figura mostra que a partir de $80 \%$ de sólidos em massa não ocorre deformação significativa do material $(0,30 \mathrm{~cm})$, não se espalhando pela superfície. 0 contrário é observado em polpas mais diluídas, cujas alturas de abatimento foram de $9,10 \mathrm{~cm}$ e $6,80 \mathrm{~cm}$, respectivamente, com $59,87 \%$ e $64,86 \%$ de sólidos em massa.

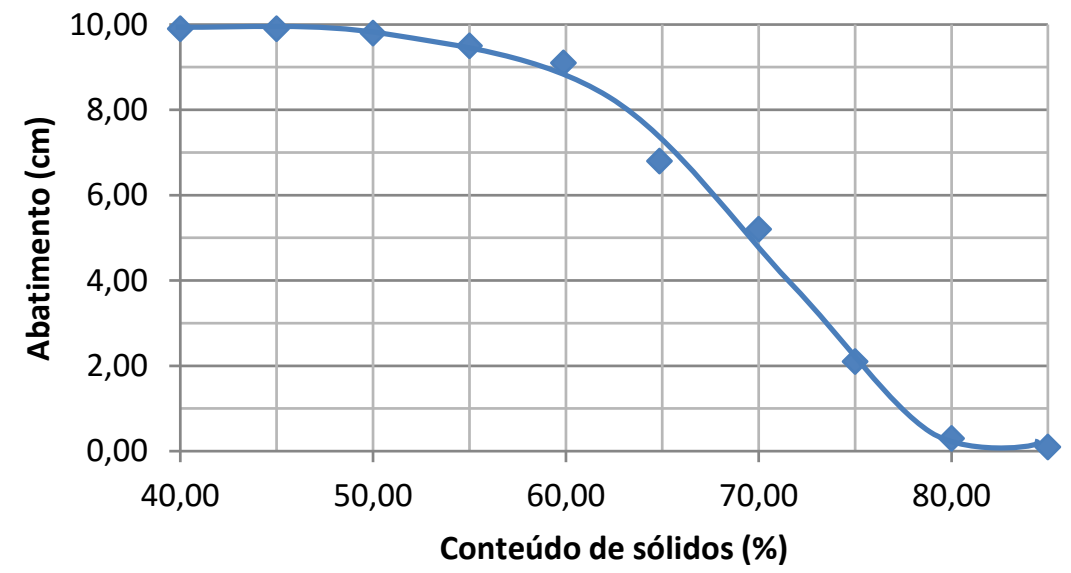

Figura 4: Abatimento de pastas minerais em função da concentração de sólidos em massa.

A Figura 6 apresenta os aspectos de espalhamentos e alturas de abatimento obtidas em diferentes concentrações de sólidos. 


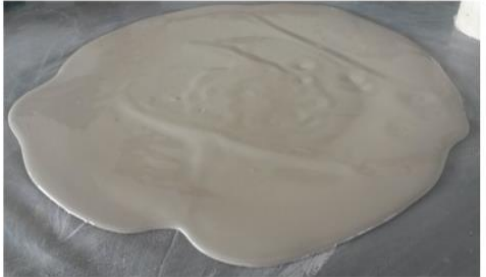

$59,87 \%$ de sólidos em massa Altura de abatimento: $9,10 \mathrm{~cm}$

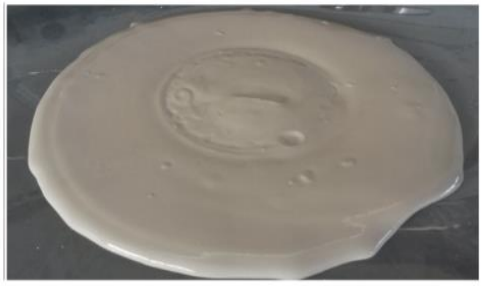

$64,86 \%$ de sólidos em massa Altura de abatimento: $6,80 \mathrm{~cm}$

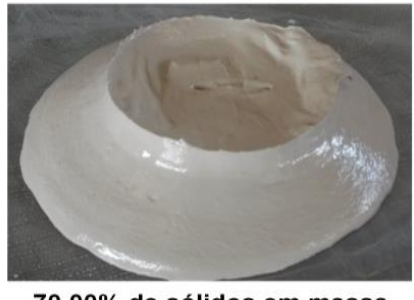

$70,00 \%$ de sólidos em massa Altura de abatimento: $5,20 \mathrm{~cm}$

Figura 5: alturas de abatimento em função da concentração de sólidos em massa.

\section{CONCLUSÕES}

Através de análise granulométrica por difração de laser, determinou-se que $50 \%$ das partículas são passantes em $6,10 \mu \mathrm{m}$, condizentes com o tamanho necessário para formação de pastas minerais. $O$ estudo do difratograma de raios $X$ mostrou que a amostra é constituída basicamente por carbonatos, tais como calcita e dolomita, embora exista uma pequena quantidade de caolinita, moscovita, quartzo e apatita.

Os melhores resultados de sedimentação descontínua em proveta ocorreram com a dosagem de $100 \mathrm{~g} / \mathrm{t}$ de poliacrilamida catiônica de alto peso molecular, cuja velocidade de sedimentação e turbidez do overflow foram, respectivamente, 2,40cm/s e 9,4 NTU.

Quanto ao abatimento de pastas minerais, a partir de $80 \%$ de sólidos em massa não se obteve deformação significativa, enquanto polpas diluídas entre 40,23 e 59,87\% de sólidos em massa, a mesma se espalhava perfeitamente pela superfície, apresentando deformação entre $9,90 \mathrm{~cm}$ e $9,10 \mathrm{~cm}$, respectivamente

\section{REFERÊNCIAS BIBLIOGRÁFICAS}

1. Araujo A.C., Souza C.C., Amarante S.C. Rejeitos adensados para disposição em superfície - nova tecnologia em minério de ferro. In: IV Simpósio Brasileiro de Minério de Ferro; 2003; Ouro Preto, Brasil. (ABM; n. 4). p. 610-619.

2. Falcucci A. A influência de floculantes poliméricos na formação de pastas minerais. [Dissertação de Mestrado]. Escola de Engenharia da Universidade Federal de Minas Gerais, Belo Horizonte; 2007.

3. Guimarães F.A.V. Revisão nos métodos de dimensionamento de espessadores e comparação dos modelos industriais. [Dissertação de Mestrado]. Escola de Engenharia da Universidade Federal de Minas Gerais, Belo Horizonte;2010.

4. Jung S.J., Biswas K. Review of current high density paste fill and its technology. Mineral Resources Engineering 2002; 11(9); 165-182.

5. Lara A.F.M. Espessamento e transporte de pasta mineral. [Monografia de Especialização]. Escola de Engenharia da Universidade Federal de Minas Gerais, Belo Horizonte; 2011.

6. Olcay R.H.B. Desenvolvimento de equipamento produtor de pasta mineral. [Dissertação de Mestrado]. Escola de Engenharia da Universidade Federal de Minas Gerais, Belo Horizonte; 2008. 
7. Sofrá F., Boger D.V. Environmental rheology for waste minimization in the minerals industry. Chemical Engineering Journal 2002; 86(6); 319-330.

8. Tão D., Parekh B.K., Honaker R. Development and pilot-scale demonstration of deep cone paste thickening process for phosphatic clay disposal. Institute of Phosphate Research 2008; 151.

9. Theriault J.A., Frostiak J., Welch D. Surface disposal of paste tailings at the Bulyanhulu Gold Mine. Internal Report 2001;1-8. 